\title{
Guidelines for writing papers
}

On the following three pages we publish instructions, guidelines, and checklists that we use, at various stages of the editorial process, to assess papers submitted to the journal. We hope that authors will note the points we look for so that the number of changes that they are required to make before we finally accept their paper may be kept to a minimum.

The BMF has agreed to accept manuscripts prepared in accordance with the Vancouver style 1 and will consider any paper that conforms to that style.

\section{Instructions to authors}

All material submitted for publication is assumed to be submitted exclusively to the BMF unless the contrary is stated. All authors must give signed consent to publication. The editor retains the customary right to style and if necessary shorten material accepted for publication.

Manuscripts will be acknowledged; letters and obituaries will not be unless a stamped addressed envelope is enclosed. Authors of letters and obituaries are not sent proofs.

All material should be typed in double spacing. Authors should give one degree and one appointment.

Original articles are usually up to 2000 words long, with no more than six tables or illustrations; they should normally report original research of relevance to clinical medicine and may appear either as Papers or as short reports. Short reports are up to 600 words long, with one table or illustration and no more than five references. Clinical case histories and brief or negative research findings may appear in this section. Papers for the Practice Observed section should cover research or any other matters relevant to primary care. Middle articles are mostly written by invitation, but we welcome reports of up to 2000 words on the organisation or assessment of medical work and on sociological aspects of medicine or the organisation, financing, and staffing of health services. Contributions for the Personal View and Materia Non Medica columns are always welcome and should contain up to 1150 and 400 words respectively. Letters should normally be of not more than 400 words, have no more than 10 references, and be signed by all authors; preference is given to those that take up points made in contributions published in the journal. Contributions to Medicine and the Media should be discussed with one of the editors before being submitted. Obituaries should not normally exceed 250 words.

Any article may be submitted to outside peer review and evaluation by the editorial committee as well as statistical assessment incorporating the use of published checklists. ${ }^{2}$ This should take four weeks but may take up to six. Manuscripts are usually published within three months of the date of final acceptance of the article.

\section{MANUSCRIPTS, TABLES, AND ILLUSTRATIONS}

Authors should keep one copy of their manuscripts for reference. All manuscripts including letters and obituaries should be typed double spaced on one side of the paper with $55 \mathrm{~cm}$ margin at the top and left hand side of the sheet. The pages should be numbered. Three copies should be submitted; if the paper is rejected these will not be returned. After being kept for three months to answer any queries they will be shredded. The authors should include their names and initials, their posts at the time they did the work, and no more than one degree each. Scientific articles should conform to the conventional structure of abstract, introduction, methods, results, discussion, and references. All papers should include a structured abstract, ${ }^{3}$ which should be no longer than 250 words. In addition, all authors should submit a paragraph up to 150 words long for the This Week in BMF page.

Drugs should be referred to by their approved, not proprietary, names, and the source of any new or experimental preparations should be given. Abbreviations should not be used. Scientific measurements should be given in SI units, but blood pressure should continue to be expressed in $\mathrm{mm} \mathrm{Hg}$.

Statistical methods should be defined in the methods section of the paper and any not in common use should be either described in detail or supported by references. General guidelines on the use of statistical methods and on the interpretation and presentation of statistical material as well as specific recommendations on statistical estimation and significance have been published. ${ }^{2}$ Whenever possible numbers of patients or subjects studied should be given. Tables and illustrations should be submitted separately from the text of the paper, and legends to illustrations should also be typed on a separate sheet. Tables should be simple and should not duplicate information in the text of the article. Illustrations should be used only when data cannot be expressed clearly in any other way. When graphs, scattergrams, or histograms are submitted the numerical data on which they are based should be supplied; in general, data given in histograms are converted into tabular form. Line drawings should be in Indian ink on heavy white paper or card, with any labelling on a separate sheet; they may also be presented as photographic prints or good quality photocopies. Other black and white illustrations should usually be prints-not negatives, transparencies, or $x$ ray films; they should be no larger than $30 \times 21 \mathrm{~cm}$ (A4) and be trimmed to remove all redundant areas; the top should be marked on the back. Staining techniques of photomicrographs should be stated. An internal scale marker should be included on the photomicrograph. Again, any labelling should be on copies, not on the prints. Patients shown in photographs should have their identity concealed or should give their written consent to publication. If any tables or illustrations submitted have been published elsewhere written consent to republication should be obtained by the author from the copyright holder (usually the publishers) and the authors.

\section{REFERENCES, PROOFS, AND REPRINTS}

References should be numbered in the order in which they appear in the text. At the end of the article the full list of references should give the names and initials of all authors (unless there are more than six, when only the first three should be given followed by et al). The authors' names are followed by the title of the article; the title of the journal abbreviated according to the style of Index Medicus (see "List of Journals Indexed," printed yearly in the January issue of Index Medicus); the year of publication; the volume number; and the first and last page numbers. References to books should give the names of any editors, place of publication, publisher, and year.

21 Soter NA, Wasserman SI, Austen KF. Cold urticaria: release into the circulation of histamine and eosinophil chemotactic factor of anaphylaxis during cold challenge. $N$ Engl f Med 1976;294:687-90.

22 Osler AG. Complement: mechanisms and functions. Englewood Cliffs: Prentice-Hall, 1976.

Information from manuscripts not yet in press, papers reported at meetings, or personal communications may be cited only in the text, not as formal references. Authors must verify references against the original documents before submitting the article. 
Manuscripts should bear the name and address of the author to whom the proofs and correspondence should be sent. Proofs are not normally sent for letters. Proof corrections should be kept to a minimum and should conform to the conventions shown in Whitaker's Almanack. If corrections need justification please include the justification in a letter, not on the proof.
Reprints are available; a scale of charges is included when a proof is sent.

1 International Committee of Medical Journal Editors. Uniform requirements for manuscripts submitted to biomedical journals. BMF 1988;296:401-5.

2 Gardner MJ, Altman DG, eds. Statistics with confidence. London: British Medical Journal, 1989

3 Haynes RB, Mulrow CD, Huth EJ, Altman DG, Gardner MJ. More informative abstracts revisited. Ann Intern Med 1990;113:69-76.

\section{Guidelines for referees}

Papers received by the journal are read first by one or more of our medical editors, who decide whether to send them to a referee; about half of all papers are sent to a referee. The referees are asked for their opinion on the originality, scientific reliability, clinical importance, and overall suitability of the paper for publication in the journal, and their reports may be sent to the authors to indicate any changes required. To help them, referees are sent a copy of our "guidelines for referees."

The $B M F$ normally sends papers to only one referee; after that any potentially acceptable papers may also be sent to our statistical adviser.

- The manuscript is a confidential document. Please do not discuss it even with the author.

- If you want to consult a colleague or junior please discuss this with us first.

- The referee is providing advice to the editors, who (aided by an editorial - "hanging"-committee) make the final decision. We will let you know our decision and will normally pass on your comments (anonymously, of course) to the author.

- Even if we do not accept a paper we would like to pass on constructive comments that might help the author to improve it.

- For this reason please give detailed comments (with references, if appropriate) that will help both the editors to make a decision on the paper and the authors to improve it. Please type your detailed comments on a separate sheet and make your recommendations and any confidential comments to the editor in a covering letter.

The broad aspects that we should like comments on include:

- Originality (truly original or known to you through foreign or specialist publications or through the grapevine); originality is our main criterion for case reports.
- Scientific reliability

-Overall design of study

- Patients studied

Adequately described and their condition defined?

- Methods

Adequately described?

Appropriate?

-Results

Relevant to problem posed?

Credible?

Well presented (including the use of tables and figures)?

-Interpretation and conclusions

Warranted by the data?

Reasonable speculation?

Is the message clear?

-References

Up to date and relevant?

Any glaring omissions?

- Importance (clinical or otherwise) of the work

- Suitability for the $B M f$ and overall recommendation

-Appropriate for general readership or more appropriate for special journal?

-If not acceptable now can the paper be made so?

- Other points

- Ethical aspects

-Need for statistical assessment

- Presentation (including writing style)

\section{Checklists for statisticians}

The comments made by referees are considered by the "hanging" committee, which decides whether each paper should be published, perhaps after revision, or rejected. If the paper seems promising the committee may decide to send it for statistical assessment. In this case a statistician looks at it, completes a checklist, and probably also writes a report, which, as with the scientific referee's report, may be sent to the author. The statisticians complete one of two checklists: one is for general papers and the other, which is more detailed, is for papers on clinical trials. For each question under the headings "Design features" and "Conduct of study/trial" the statistician is asked to circle the reply Yes, Unclear, or No; for each question under the headings "Analysis" and "Recommendations" he or she is asked to circle the reply Yes or No.

CHECKLIST FOR STATISTICAL REVIEW OF GENERAL PAPERS

Design features

1 Was the objective of the study sufficiently described?

2 Was an appropriate study design used to achieve the objective?

3 Was there a satisfactory statement given of source of subjects?

4 Was a pre-study calculation of required sample size reported?

Conduct of study

5 Was a satisfactory response rate achieved?

Analysis and presentation

6 Was there a statement adequately describing or referencing all statistical procedures used?

7 Were the statistical analyses used appropriate?
8 Was the presentation of statistical material satisfactory?

9 Were the confidence intervals given for the main results?

10 Was the conclusion drawn from the statistical analysis justified?

\section{Recommendation on paper}

11 Is the paper of acceptable statistical standard for publication?

12 If "No" to question 10, could it become acceptable with suitable revision?

CHECKLIST FOR STATISTICAL REVIEW OF PAPERS ON CLINICAL TRIALS

Design features

1 Was the objective of the trial sufficiently described?

2 Was a satisfactory statement given of diagnostic criteria for entry to the trial? 
3 Was there a satisfactory statement given of source of subjects?

4 Were concurrent controls used (as opposed to historical controls)?

5 Were the treatments well defined?

6 Was random allocation to treatment used?

7 Was the method of randomisation described?

8 Was there an acceptably short delay from allocation to start of treatment?

9 Was the potential degree of blindness used?

10 Was there a satisfactory statement of criteria for outcome measures?

11 Were the outcome measures appropriate?

12 Was a pre-study calculation of required sample size reported?

13 Was the duration of post-treatment follow up stated?

\section{Conduct of trial}

14 Were the treatment and control groups comparable in relevant measures?

15 Were a high proportion of the subjects followed up?
16 Did a high proportion of subjects complete treatment?

17 Were the drop outs described by treatment/control groups?

18 Were side effects of treatment reported?

\section{Analysis and presentation}

19 Was there a statement adequately describing or referencing all statistical procedures used?

20 Were the statistical analyses used appropriate?

21 Were prognostic factors adequately considered?

22 Was the presentation of statistical material satisfactory?

23 Were confidence intervals given for the main results?

24 Was the conclusion drawn from the statistical analysis justified?

Recommendation on paper

25 Is the paper of acceptable statistical standard for publication?

26 If "No" to question 25, could it become acceptable with suitable revision?

\section{Adverse drug reactions checklist}

*Based on guidelines drawn up at a workshop of representatives of the pharmaceutical industry, department of clinical pharmacology, drug regulating agencies, medical and scientific editors, and science correspondents of the general press in 1984 (Ciba-Geigy workshop; BMT 1984;289:898).
When we receive a report of an adverse drug reaction we usually send the author a checklist of points that should be mentioned in such reports.

It is our policy to ask authors who are reporting side effects of drugs to contact the Committee on the Safety of Medicines and the manufacturer of the drug to inquire if they have had similar reports and to let us have sight of their replies.

Case reports of adverse drug reactions should include the following information ${ }^{\star}$ :

1 Birth date or age and sex

2 Suspected drug and all drugs currently being taken:

Start, stop, and restart dates

Dose

Indication for drug treatment

3 Timing of suspected adverse drug reaction in relation to drugs taken and outcome.

4 Other diseases, environmental factors, and timing.

5 Prior experience with drug or adverse reactions to related drug
6 Ancillary information from pharmaceutical company and regulatory agency

7 Any published reports?

8 Other factors relevant to verify specific types of adverse drug reactions (for example, blood concentration in overdose, baseline laboratory data, ethnic group)

Any report that describes a series of cases should provide the following information ${ }^{\star}$ :

1 Age and sex

2 Number of patients treated

3 Number with adverse drug reactions

4 Number of events

Generally something more than simple coincidence in time is required: rechallenge (with the patient's informed consent) or immunological investigations may tip the balance of probabilities.

\section{Technical editor's checklist}

Finally, one of our technical editors looks at the accepted papers, either before they go back to the authors for revision according to the reviewers' remarks or before the letter of acceptance is sent out if the paper does not need revision. The technical editor indicates various details to be corrected or supplied.

When returning your revised paper please supply the information requested below.

1 Summary of 150 words for "This Week in the BMJ."

2 Structured abstract (details attached)

3 One degree for each author and one position held at the time of the study.

4 Author for correspondence.

5 All values in SI units (except for blood pressure in $\mathrm{mm} \mathrm{Hg}$ ).

6 Actual numbers of patients/subjects, as well as percentages, within the text and tables.

7 The actual data from which histograms were drawn (if these are percentages, please also provide the actual numbers). We generally convert histograms into tables, but even if we leave them as histograms the data from which they were drawn are helpful.

8 This article is too long as a short report. It must be reduced to within 600 words with one table or figure and at most five references.
9 Abbreviations should not be used and should be spelt out in full each time.

10 Please type text and references in double spacing 11 References must be set out in Vancouver style (BMF 1988;296:401-5). Please provide:

(a) the surnames and initials of all authors (or of only the first three if there are more than six); (ref )

(b) the title of the article or chapters; (ref )

(c) the final page numbers of each article; (ref )

(d) the editors of books; (ref )

(e) the publisher of each book; (ref )

$(f)$ the place of publication of books; (ref )

$(g)$ the year of publication of books; (ref )

(h) the title of the journal in full; (ref )

(i) has the reference been published? If not please cite in text and renumber other references; (ref . ) 